\title{
The effect of glucocorticoids on serum cystatin C in identifying acute kidney injury: a propensity-matched cohort study
}

Silin Liang ${ }^{1,2,3 \dagger}{ }^{\text {, Mai Shi }}{ }^{4 \dagger}$, Yunpeng Bai ${ }^{5,6 \dagger}$, Yujun Deng ${ }^{2}$, Miaoxian Fang ${ }^{1}$, Jiaxin Li ${ }^{1}$, Yijin Wu ${ }^{1}$, Wenying Peng ${ }^{1}$, Yating $\mathrm{Hou}^{7}$, Heng Fang ${ }^{1,2}$, Huidan Zhang ${ }^{1,2}$ and Chunbo Chen ${ }^{1,2,3^{*}}$

\begin{abstract}
Background: Glucocorticoids may impact the accuracy of serum cystatin C (sCysC) in reflecting renal function. We aimed to assess the effect of glucocorticoids on the performance of sCysC in detecting acute kidney injury (AKI) in critically ill patients.

Methods: A prospective observational cohort study was performed in a general intensive care unit (ICU). Using propensity score matching, we successfully matched 240 glucocorticoid users with 960 non-users among 2716 patients. Serum creatinine (SCr) and sCysC were measured for all patients at ICU admission. Patients were divided into four groups based on cumulative doses of glucocorticoids within 5 days before ICU admission (Group I: nonusers; Group II: 0 mg < prednisone $\leq 50$ mg; Group III: 50 mg < prednisone $\leq 150$ mg; Group IV: prednisone > 150 $\mathrm{mg}$ ). We compared the performance of sCysC for diagnosing and predicting AKI in different groups using the area under the receiver operator characteristic curve (AUC).

Results: A total of 240 patients received glucocorticoid medication within 5 days before ICU admission. Before and after matching, the differences of sCysC levels between glucocorticoid users and non-users were both significant $(P<0.001)$. The multiple linear regression analysis revealed that glucocorticoids were independently associated with sCysC $(P<0.001)$. After matching, the group I had significantly lower sCysC levels than the group III and group IV $(P<0.05)$, but there were no significant differences in sCysC levels within different glucocorticoids recipient groups $(P>0.05)$. Simultaneously, we did not find significant differences in the AUC between any two groups in the matched cohort $(P>0.05)$.
\end{abstract}

Conclusions: Glucocorticoids did not impact the performance of sCysC in identifying AKI in critically ill patients.

Keywords: Acute kidney injury, Cystatin C, Intensive care unit, Renal biomarker

\footnotetext{
* Correspondence: gghccm@163.com

†Silin Liang, Mai Shi and Yunpeng Bai contributed equally to this study, Cofirst author.

'Department of Intensive Care Unit of Cardiovascular Surgery, Guangdong Cardiovascular Institute, Guangdong Provincial People's Hospital, Guangdong Academy of Medical Sciences, 96 Dongchuan Road, Guangzhou 510080, Guangdong Province, People's Republic of China

${ }^{2}$ Department of Critical Care Medicine, Guangdong Provincial People's

Hospital, Guangdong Academy of Medical Sciences, 106 Zhongshan Er Road, Guangzhou 510080, Guangdong Province, People's Republic of China

Full list of author information is available at the end of the article
}

(C) The Author(s). 2020 Open Access This article is licensed under a Creative Commons Attribution 4.0 International License, which permits use, sharing, adaptation, distribution and reproduction in any medium or format, as long as you give appropriate credit to the original author(s) and the source, provide a link to the Creative Commons licence, and indicate if changes were made. The images or other third party material in this article are included in the article's Creative Commons licence, unless indicated otherwise in a credit line to the material. If material is not included in the article's Creative Commons licence and your intended use is not permitted by statutory regulation or exceeds the permitted use, you will need to obtain permission directly from the copyright holder. To view a copy of this licence, visit http://creativecommons.org/licenses/by/4.0/ The Creative Commons Public Domain Dedication waiver (http://creativecommons.org/publicdomain/zero/1.0/) applies to the data made available in this article, unless otherwise stated in a credit line to the data. 


\section{Key messages}

- Glucocorticoids could induce an increase in sCysC levels. However, the relationship between the sCysC and glucocorticoids was not manifested in a dose-dependent manner.

- Glucocorticoids did not have a statistically significant effect on the performance of $\mathrm{sCysC}$ in detecting AKI in critically ill patients.

\section{Background}

Acute kidney injury (AKI) is an alarming burden in critically ill patients with a high incidence and independently impacts prognosis [1-4]. The therapeutic outcome of AKI is unsatisfactory as the initiation of effective treatments are started relatively late after a noticeable elevation in serum creatinine $(\mathrm{SCr})[5,6]$. In this context, the exploration of an early biomarker could significantly improve the prognosis of AKI.

Cystatin $\mathrm{C}(\mathrm{CysC})$, a cysteine proteinase inhibitor, is freely filtered by the glomerulus and entirely reabsorbed by the proximal tubules [7-9]. Contrast to $\mathrm{SCr}$, the concentrations of serum cystatin $\mathrm{C}(\mathrm{sCys} \mathrm{C})$ show a small individual variability as nonrenal factors only slightly influenced it $[8,9]$.. Therefore, sCysC may be superior to $\mathrm{SCr}$ in detecting a minimal change in the glomerular filtration rate (GFR) [7, 10, 11]. Previous studies showed that it might be a potential biomarker for the early detection of AKI [12-16].

However, as $\mathrm{sCysC}$ is widely used in clinical application, many studies reported other factors that might interfere with its concentrations beyond renal function, such as glucocorticoid therapy, thyroid function, and glycemic status [17-22]. CysC is produced by all nucleated cells in the human body at a relatively constant rate $[7,8]$. However, it was shown that glucocorticoids could stimulate the production of $\mathrm{CysC}$ in vitro [23]. Moreover, glucocorticoid medication would also increase $\mathrm{sCysC}$ concentrations in experimental animals, such as rats and dogs [24, 25]. Glucocorticoids have extensive applications in human medicine because of its functions of anti-inflammation and immunosuppression. Many studies already suggested that glucocorticoids could impact $\mathrm{sCysC}$ concentrations independently of renal function in patients [26-29]. However, it remains undefined whether glucocorticoids influence $\mathrm{s} C y \mathrm{SC}$ to detect $\mathrm{AKI}$ in critically ill patients.

Therefore, this prospective, observational, propensitymatched study was performed in a large critically ill cohort to illuminate whether glucocorticoids impacted the diagnostic and predictive accuracy of $\mathrm{sCysC}$ in detecting AKI.

\section{Methods}

\section{Study design and participants}

This prospective observational study was undertaken in the general intensive care unit (ICU) of Guangdong Provincial People's Hospital from October 2014 to December 2017.
Patients aged 18 years or older admitted to ICU were enrolled for the study. The exclusion criteria incorporated preexisting Cushing syndrome or adrenocortical hypofunction, end-stage renal disease (ESRD) or undergoing renal replacement therapy (RRT) before admission, renal transplantation or nephrectomy, missing clinical data or refusal of consent. The primary outcome of this study was the diagnosis of AKI within 1 week after ICU admission, and the secondary outcomes included length of ICU and hospital stay, as well as ICU and hospital mortality. The protocol was followed according to that of the Strengthening the Reporting of Observational Studies in Epidemiology [30] and Standards for Reporting Diagnostic Accuracy [31] criteria. The current study received the approval of ethics committee of the Guangdong Provincial People's Hospital and all methods were performed in accordance with the relevant guidelines and regulations. Written informed consent was obtained from each participant or a family member at the time of enrollment.

\section{Data collection}

We prospectively collected patients' baseline clinical data. All serum samples for determining $\mathrm{SCr}, \mathrm{sCysC}$, and serum albumin were collected concurrently within $1 \mathrm{~h}$ after ICU admission. $\mathrm{SCr}$ was measured at least once a day as a part of routine clinical care during ICU hospitalization. The following demographic and clinical characteristics were collected: age, sex, body mass index (BMI), preexisting clinical conditions, previous glucocorticoid administration within 5 days before entering ICU, admission type, baseline $\mathrm{SCr}$, baseline-estimated glomerular filtration rate (eGFR), $\mathrm{SCr}$ at ICU admission, serum albumin and Acute Physiology and Chronic Health Evaluation II (APACHE II) score at ICU admission. The outcome variables were documented, including the occurrence of AKI within 1 week after ICU admission, length of ICU stay, length of hospital stay, ICU mortality, and in-hospital mortality. We calculated the eGFR based on the Chronic Kidney Disease Epidemiology Collaboration (CKD-EPI) creatinine equation [32].

\section{Definitions}

AKI was diagnosed according to the Kidney Disease Improving Global Outcomes (KDIGO) criteria as any of the following: increase in SCr by $0.3 \mathrm{mg} / \mathrm{dl}(26.5 \mathrm{mmol} / \mathrm{l})$ within $48 \mathrm{~h}$, increase in SCr to 1.5 times of the baseline level within 1 week, or urine output $<0.5 \mathrm{~mL} / \mathrm{kg} / \mathrm{h}$ for 6 $h$ [33]. Because of insufficient sensitivity of urine output in diuretics administration, we diagnosed AKI based on $\mathrm{SCr}$ [34]. We determined the baseline SCr according to the following rules ranked in descending order of preference [35]: (1) the most recent pre-ICU value between 30 and 365 days before ICU admission; (2) a stable pre-ICU value $>365$ days for patients aged $<40$ years (stable defined as within $15 \%$ of the lowest ICU measurement) 
before ICU admission; (3) pre-ICU value $>365$ days before ICU admission and less than the initial SCr at ICU admission; (4) a pre-ICU value (between 3 and 39 days before ICU admission) less than or equal to the initial onadmission SCr to ICU and not distinctly during AKI; (5) the lowest $\mathrm{SCr}$ upon initial admission to ICU, the last ICU value, or the minimum value at follow-up to 365 days. Established AKI and later-onset AKI were diagnosed if patients reached the KDIGO criteria at ICU admission or within 1 week after ICU admission, respectively. Mild AKI was defined as KDIGO stage 1 and severe AKI was defined as stage 2 or stage 3 of KDIGO criteria [33, 36].

\section{Patient groups}

Thus far, previous studies showed that there was a significant correlation between sCysC levels and glucocorticoids in the first 5 days after the administration of glucocorticoids $[28,29]$. Therefore, the patients were divided into four groups based on accumulated doses of glucocorticoids within 5 days before admission to ICU: Group I (nonusers), Group II (0 $\mathrm{mg}<$ prednisone $\leq 50 \mathrm{mg}$ ), Group III (50 $\mathrm{mg}<$ prednisone $\leq 150 \mathrm{mg}$ ), Group IV (prednisone $>150$ $\mathrm{mg}$ ). As attested by potency and duration of action of glucocorticoids, we used this dosage calculation, $4 \mathrm{mg} /$ day of methylprednisolone and $0.75 \mathrm{mg} /$ day of dexamethasone was equal to a dosage of $5 \mathrm{mg} /$ day of prednisone [37].

\section{Laboratory methods}

All samples were measured within $24 \mathrm{~h}$ after collection at the central laboratory of the Guangdong Provincial People's Hospital. SCr, sCysC, and serum albumin were measured using a UniCel DxC 800 Synchron System (Beckman Coulter, CA, USA). sCysC was measured by immunoturbidimetry, the coefficients of inter-assay and intraassay variations for which were $\leq 5 \%$ and $\leq 10 \%$, respectively.

\section{Statistical analysis}

To avoid potential confounders and selection biases caused by nonrandomized in this study, we used the propensity score matching. We generated the propensity score for each patient according to the probability of receiving glucocorticoids produced by multivariable logistic regression analysis model. The multivariable logistic regression analysis model $(P<0.001$; Hosmer and Lemeshow goodnessof-fit test, $P=0.935$ ) obtained the following covariates: age, sex, BMI, APACHE II score, serum albumin, baseline eGFR, diabetes mellitus (DM), hypertension, chronic kidney disease (CKD), coronary heart disease (CHD), chronic heart failure (CHF), stroke, malignancy, chronic obstructive pulmonary disease (COPD), chronic liver disease, admission type, and grade of AKI. And then, we used the propensity score to match glucocorticoid users with non-users at a ratio of 1:4 using nearest neighbor matching. Patients without a corresponding match were excluded. After all propensity score matches were completed, we evaluated the balance in baseline variates. Propensity score matching was performed using SPSS version 23.0 (SPSS, USA, IL).

A two-tailed $P$ value $<0.05$ was considered statistically significant. All continuous variables did not follow normal distribution as tested by SPSS software. Continuous variables were reported as median (interquartile range, IQR) and compared using the Wilcoxon rank-sum test. Categorical variables were reported as absolute value (percentage) and compared using the chi-square test or Fisher's exact test. The bivariate correlation analysis was used to assess the association between two variables. Multivariable linear regression analysis with a stepwise variable selection was also used to assess the relationship between sCysC and other variables. A receiver operating characteristic (ROC) curve analysis was generated, and the area under the curve (AUC) in each group as assessed using the Hanley-McNeil method, and the optimal cutoff value for AKI detection was determined with the Youden's index. All statistical analyses were performed using SPSS version 23.0 (SPSS, IL, USA) and MedCalc version 18.2.1 (MedCalc Software, Ostend, Belgium) software programs.

\section{Results}

\section{Patient characteristics and outcomes}

There were 2716 patients got engaged in this study (Fig. 1). A total of 166 patients were excluded. Glucocorticoids were prescribed for $8.8 \%(240 / 2716)$ of patients. In total, $23.3 \%$ of patients $(634 / 2716)$ met the diagnostic criteria of AKI, including patients accounted for $34.9 \%$ (221/634) occurred established AKI, and patients accounted for $65.1 \%$ (413/634) developed later-onset AKI.

Table 1 shows the characteristics of the patients. Compared with patients who were not prescribed glucocorticoids, users were more likely to have a higher APACHE II score (11 vs. $9, P<0.001$ ), and had a significantly higher percentage of comorbidities, including malignancy $(27.9 \%$ vs. $14.7 \%, P<0.001)$, COPD (5.4\% vs. $1.8 \%, P<0.001)$ and chronic liver disease $(2.9 \%$ vs $1.0 \%, P=0.017)$. Moreover, there was a significant difference between glucocorticoid users and non-users in admission type. Patients using glucocorticoids were more likely to suffer from more severe AKI.

\section{Factors associated with $\mathrm{sCysC}$}

The results of bivariate correlations analysis indicated a significant, but weak, correlation between $\mathrm{sCysC}$ and previous use of glucocorticoids (correlation coefficient $=0.074$, $P<0.001$ ) (Table 2). In multivariable linear regression analysis (Table 3), we found the same association between sCysC and previous use of glucocorticoids (standardized $\beta=0.092, P=0.003$ ). There was also an association between the cumulative dose of steroids and cystatin $C$ in the above two types of correlation analysis. Besides, the correlation and regression analyses demonstrated that other factors 


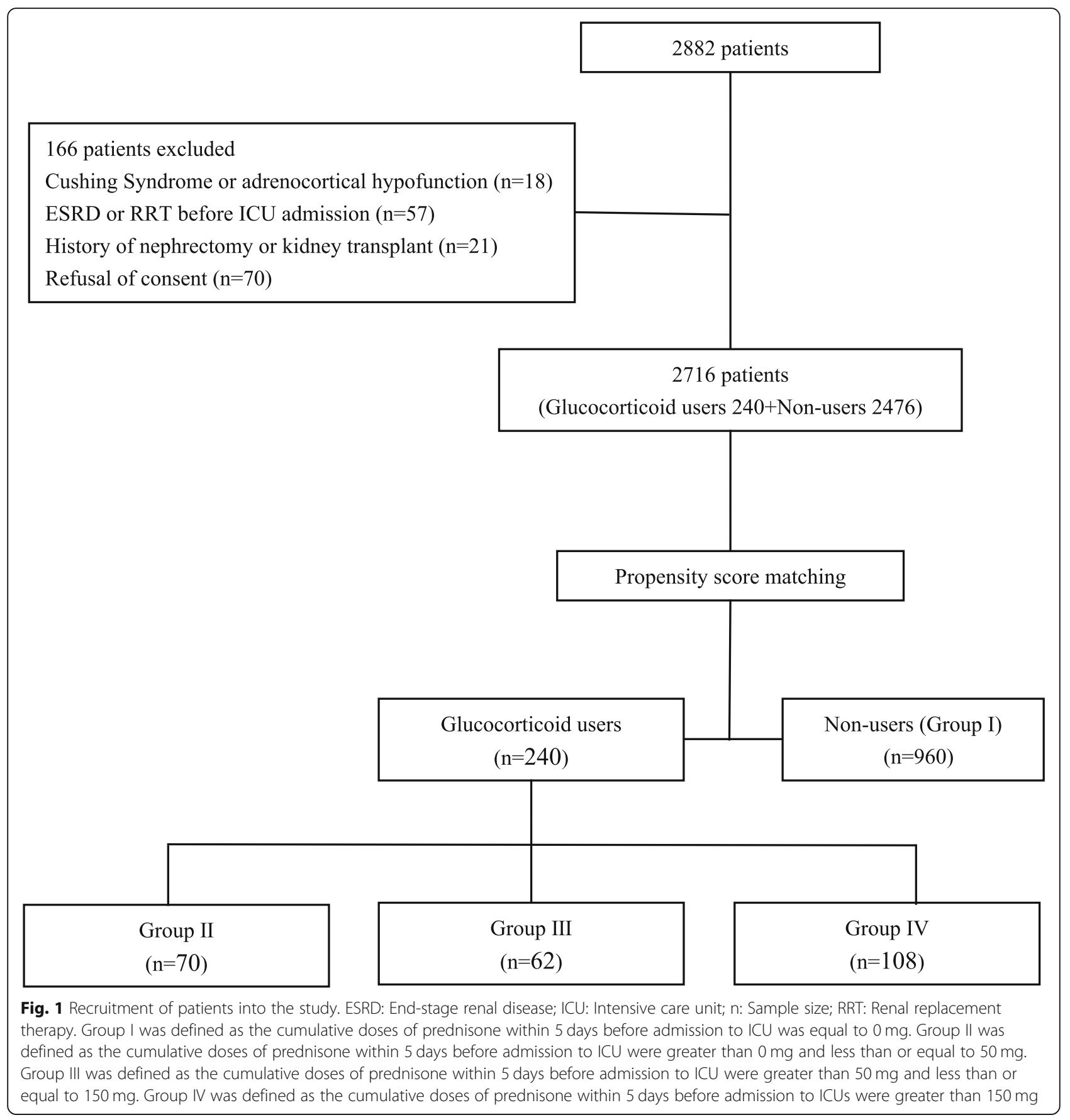

also were associated with sCysC. Therefore, we used the propensity score matching to eliminate their impacts.

\section{Propensity score match}

Based on the propensity score of each patient, 240 glucocorticoid users were successfully matched to 960 nonusers (Table 1). There were no significant differences in the baseline characteristics between the matched glucocorticoid users and non-users.
The concentrations of $\mathrm{sCys} C$ in different groups

We found a significant difference in $\mathrm{sCysC}$ levels between glucocorticoid users and non-users $(0.940 \mathrm{mg} / \mathrm{L}$ vs $0.810 \mathrm{mg} / \mathrm{L} ; P<0.001$ ), although $\mathrm{sCysC}$ levels were elevated moderately (Fig. 2). Before matching, sCysC levels were statistically significantly lower in group I than the other three groups $(P<0.05)$, and the $\mathrm{sCysC}$ levels of different glucocorticoids recipient groups were not different $(P>0.05)$. After matching, there were the same results in the different glucocorticoids recipient groups 
Table 1 Baseline data and outcomes

\begin{tabular}{|c|c|c|c|c|c|c|}
\hline \multirow[t]{2}{*}{ Variables } & \multicolumn{3}{|l|}{ Before matching } & \multicolumn{3}{|l|}{ After matching } \\
\hline & $\begin{array}{l}\text { Corticosteroid } \\
\text { users }(n=240)\end{array}$ & $\begin{array}{l}\text { Non-users } \\
(n=2476)\end{array}$ & $P$ value & $\begin{array}{l}\text { Corticosteroid } \\
\text { users }(n=240)\end{array}$ & $\begin{array}{l}\text { Non-users } \\
(n=960)\end{array}$ & $P$ value \\
\hline Cumulative dose of prednisone, mg & $133.3(50.0-300.0)$ & 0 & & $133.3(50.0-300.0)$ & 0 & \\
\hline \multicolumn{7}{|l|}{ Baseline characteristics } \\
\hline Age, years & $55.0(44.0-65.0)$ & $55.0(43.0-65.0)$ & 0.949 & $55.0(44.0-65.0)$ & $54.0(40.0-67.0)$ & 0.800 \\
\hline Male sex, n (\%) & $158(65.8)$ & $1280(51.7)$ & $<0.001$ & $158(65.8)$ & $615(64.1)$ & 0.608 \\
\hline $\mathrm{BMI}, \mathrm{kg} / \mathrm{m}^{2}$ & $22.46(20.77-24.95)$ & $22.32(20.70-24.44)$ & 0.506 & $22.46(20.77-24.95)$ & $22.45(20.95-24.46)$ & 0.986 \\
\hline \multicolumn{7}{|l|}{ Preexisting clinical conditions } \\
\hline Hypertension, n (\%) & $46(19.2)$ & $476(19.2)$ & 0.983 & $46(19.2)$ & $177(18.4)$ & 0.795 \\
\hline $\mathrm{DM}, \mathrm{n}(\%)$ & $21(8.8)$ & $208(8.4)$ & 0.852 & $21(8.8)$ & $82(8.5)$ & 0.918 \\
\hline CKD, n (\%) & $12(5.0)$ & $90(3.6)$ & 0.288 & $12(5.0)$ & $45(4.7)$ & 0.839 \\
\hline CHD, n (\%) & $8(3.3)$ & $94(3.8)$ & 0.719 & $8(3.3)$ & $29(3.0)$ & 0.802 \\
\hline Stroke, n (\%) & $24(10.0)$ & $326(13.2)$ & 0.162 & $24(10.0)$ & $106(11.0)$ & 0.642 \\
\hline $\mathrm{CHF}, \mathrm{n}(\%)$ & $8(3.3)$ & $62(2.5)$ & 0.439 & $8(3.3)$ & $27(2.8)$ & 0.668 \\
\hline Malignancy, n (\%) & $67(27.9)$ & $364(14.7)$ & $<0.001$ & $67(27.9)$ & $256(26.7)$ & 0.696 \\
\hline COPD, n (\%) & $13(5.4)$ & $45(1.8)$ & $<0.001$ & $13(5.4)$ & $35(3.6)$ & 0.211 \\
\hline Chronic liver disease, n (\%) & $7(2.9)$ & $24(1.0)$ & 0.017 & $7(2.9)$ & $19(2.0)$ & 0.372 \\
\hline Admission type & & & $<0.001$ & & & 0.258 \\
\hline Elective surgical, n (\%) & $155(64.6)$ & $1887(76.2)$ & & $155(64.6)$ & $650(67.7)$ & \\
\hline Emergency surgical, n (\%) & $17(7.1)$ & $205(8.3)$ & & $17(7.1)$ & $84(8.8)$ & \\
\hline Medical, n (\%) & $68(28.3)$ & $384(15.5)$ & & $68(28.3)$ & $226(23.5)$ & \\
\hline Baseline eGFR, ml/min/1.73 m² & $103.70(89.81-115.39)$ & $100.23(85.83-112.60)$ & 0.036 & $103.70(89.81-115.39)$ & $103.19(88.62-116.34)$ & 0.875 \\
\hline Baseline SCr, umol/L & $63.85(54.00-76.53)$ & $65.00(53.36-79.69)$ & 0.639 & $63.85(54.00-76.53)$ & $64.00(51.60-79.50)$ & 0.742 \\
\hline $\mathrm{SCr}$ at ICU admission umol/L & $76.17(64.00-92.15)$ & 73.00 (59.40-91.80) & 0.056 & 76.17 (64.00-92.15) & $76.00(60.03-96.00)$ & 0.708 \\
\hline Serum albumin, $\mathrm{g} / \mathrm{L}$ & $30.45(26.60-33.80)$ & $31.60(27.50-35.00)$ & 0.001 & $30.45(26.60-33.80)$ & $30.30(26.36-34.00)$ & 0.999 \\
\hline APACHE I| score & $11(7.0-18.0)$ & $9(6.0-14.0)$ & $<0.001$ & $11.0(7.0-18.0)$ & $11(7.0-16.0)$ & 0.537 \\
\hline \multicolumn{7}{|l|}{ Primary outcomes } \\
\hline Total AKI, n (\%) & $88(36.7)$ & $546(22.1)$ & $<0.001$ & $88(36.7)$ & $334(34.8)$ & 0.586 \\
\hline Established AKI, n (\%) & $29(12.1)$ & $192(7.8)$ & 0.019 & $29(12.1)$ & $128(13.3)$ & 0.608 \\
\hline Later-onset AKI, n (\%) & $59(24.6)$ & $354(14.3)$ & $<0.001$ & $59(24.6)$ & $206(21.5)$ & 0.297 \\
\hline Grade of AKI, & & & $<0.001$ & & & 0.486 \\
\hline Non-AKI, n (\%) & $152(63.3)$ & $1930(77.9)$ & & $152(63.3)$ & $626(65.2)$ & \\
\hline Mild AKI, n (\%) & $50(20.8)$ & $351(14.2)$ & & $50(20.8)$ & $205(21.4)$ & \\
\hline Severe AKI, n (\%) & 38 (15.8) & $195(7.9)$ & & 38 (15.8) & $129(13.4)$ & \\
\hline
\end{tabular}

The non-normally distributed continuous variables are expressed as median (25th percentile to 75 th percentile [interquartile range]). Categorical variables are expressed as $\mathrm{n}(\%)$

APACHE II score Acute Physiology and Chronic Health Evaluation II score; AKI Acute kidney injury; BMI Body mass index; CKD Chronic kidney disease; CHD Coronary heart disease; CHF Chronic heart failure; COPD Chronic obstructive pulmonary disease; DM Diabetes mellitus; eGFR Estimated glomerular filtration rate; ICU Intensive care unit; KDIGO Kidney Disease: Improving Global Outcomes; $n$ Sample size; SCr Serum creatinine; Established AKI, defined as diagnosis of AKI at ICU admission; Later-onset AKI, indicated no AKI diagnosis at ICU admission but reaching the KDIGO criteria within 1 week after admission; Mild-AKI: defined as reaching KDIGO stage 1 diagnostic criteria of AKI; Severe-AKI, defined as reaching KDIGO stage 2 or stage 3 diagnostic criteria of AKI $P$ value for global comparisons among groups by rank sum test and chi-square test for continuous and categorical variables, respectively 
Table 2 Factors associated with sCysC using bivariate correlation analysis

\begin{tabular}{|c|c|c|}
\hline Variables & Correlation coefficient & $P$ \\
\hline \multicolumn{3}{|l|}{ Spearman method } \\
\hline Age, years & 0.391 & $<0.001$ \\
\hline $\mathrm{BMl}, \mathrm{kg} / \mathrm{m}^{2}$ & 0.029 & 0.134 \\
\hline $\begin{array}{l}\text { Baseline eGFR, } \\
\mathrm{ml} / \text { minute } / 1.73 \mathrm{~m}^{2}\end{array}$ & -0.477 & $<0.001$ \\
\hline Baseline SCr, umol/L & 0.438 & $<0.001$ \\
\hline $\begin{array}{l}\text { SCr at ICU admission, } \\
\text { umol/L }\end{array}$ & 0.569 & $<0.001$ \\
\hline $\begin{array}{l}\text { Cumulative dose of } \\
\text { prednisone, mg }\end{array}$ & 0.152 & $<0.001$ \\
\hline Serum albumin, g/L & -0.118 & $<0.001$ \\
\hline APACHE II score & 0.397 & $<0.001$ \\
\hline Grade of AKI, n (\%) & 0.414 & $<0.001$ \\
\hline \multicolumn{3}{|l|}{ Point-biserial method } \\
\hline Male sex, n (\%) & 0.164 & $<0.001$ \\
\hline $\begin{array}{l}\text { Previous use of } \\
\text { glucocorticoids, n (\%) }\end{array}$ & 0.074 & $<0.001$ \\
\hline Hypertension, n (\%) & 0.255 & $<0.001$ \\
\hline $\mathrm{DM}, \mathrm{n}(\%)$ & 0.182 & $<0.001$ \\
\hline CKD, n (\%) & 0.473 & $<0.001$ \\
\hline CHD, n (\%) & 0.160 & $<0.001$ \\
\hline Stroke, n (\%) & 0.108 & $<0.001$ \\
\hline CHF, n (\%) & 0.207 & $<0.001$ \\
\hline Malignancy, n (\%) & -0.003 & 0.889 \\
\hline COPD, n (\%) & 0.111 & $<0.001$ \\
\hline Chronic Liver disease, n (\%) & 0.043 & 0.026 \\
\hline
\end{tabular}

APACHE II score Acute Physiology and Chronic Health Evaluation II score; AKI Acute kidney injury; BMI Body mass index; $C K D$ Chronic kidney disease; $C H D$ Coronary heart disease; CHF Chronic heart failure; COPD Chronic obstructive pulmonary disease; DM Diabetes mellitus; eGFR Estimated glomerular filtration rate; ICU Intensive care unit; KDIGO Kidney Disease: Improving Global Outcomes; $n$ Sample size; $S C r$ Serum creatinine; Mild-AKI: defined as reaching KDIGO stage 1 diagnostic criteria of AKI; Severe-AKI, defined as reaching $\mathrm{KDIGO}$ stage 2 or stage 3 diagnostic criteria of AKI

$(P>0.05)$, but the sCysC levels of group I were significantly lower than the $\mathrm{sCysC}$ levels in group III or group IV $(P<0.05)$ (Fig. 3).

\section{Detection of AKI by sCysC concerning different glucocorticoids doses}

Table 4 shows the AUC-ROC analysis for the performance of sCysC to detect AKI in different groups. Before matching, the AUC calculated by sCysC for the entire cohort was $0.769,0.810$, and 0.748 for total AKI, established AKI, and later-onset AKI respectively (Additional file 1: Fig. S1). After matching, the AUC calculated by sCysC for the entire cohort was $0.758,0.802$, and 0.732 for detecting total AKI, established AKI, and later-onset AKI respectively (Additional file 1: Fig. S1).
The patients were split into four groups based on whether they received glucocorticoid therapy and the accumulated doses of glucocorticoids within 5 days before ICU admission. Before matching, the AUC for sCysC in detecting total AKI was 0.769, 0.733, 0.736 and 0.724 in group I, group II, group III, and group IV respectively. The AUC for sCysC in diagnosing established AKI was $0.810,0.760,0.781$, and 0.779 in group I, group II, group III, and group IV respectively. The AUC for sCysC in predicting later-onset AKI was 0.747, 0.714, 0.712, 0.707 in group I, group II, group III, and group IV respectively. After matching, the AUC for $\mathrm{sCysC}$ in detecting total AKI, established AKI, and later-onset AKI was 0.768, 0.812 , and 0.740 respectively in group I; $0.733,0.760$, and 0.714 respectively in group II; $0.736,0.781$, and 0.712 respectively in group III; $0.724,0.779$, and 0.707 respectively in group IV. In summary, we did not observe a significant difference between AUC in any two groups for total AKI, established AKI, and later-onset AKI before and after the matching. Before and after matching, the cutoff values of sCysC in detecting AKI were different in diverse glucocorticoids recipient groups, but the change of cutoff values of sCysC were irregular.

\section{Discussion}

In this prospective, propensity-matched cohort study, we evaluated whether glucocorticoids affect the ability of sCysC to detect AKI in critically ill patients. We adopted a propensity score matching to eliminate the influences of potential confounding factors. Before and after matching, we found that the abilities of $\mathrm{sCysC}$ in detecting AKI were not different among patients with or without glucocorticoid therapy.

$\mathrm{sCysC}$ has an advantage that it might be less influenced by nonrenal factors than SCr $[8,9]$. However, many studies have shown conflicting results. These studies suggested that the relationship between GFR and sCysC might have a potential variability due to some nonrenal factors, including age, sex, thyroid hormones, glycemic status, hepatic impairment, as well as glucocorticoids [17-22, 26-29, 38-43]. Bjarnadottir et al. demonstrated in vitro that dexamethasone could lead to $\mathrm{CysC}$ level increase by the dose-dependent manner [23]. Recent studies confirmed this in animals [24, 25], and we found similar results in our current study. We found that $\mathrm{sCysC}$ level in glucocorticoid users was higher than that in non-users, no matter with or without matching. This phenomenon might be caused by glucocorticoids increasing the transcription of the CysC by induction of the promoter [23]. Alternatively, glucocorticoids have a nephrotoxic effect as its ability of causing metabolic alkalosis and hypertension [44]. This may be another reason why glucocorticoids could lead to an increase in the $\mathrm{sCysC}$ level. 
Table 3 Factors associated with sCysC using multivariate linear regression analysis

\begin{tabular}{lll}
\hline Independent variables & \multicolumn{2}{l}{ sCysC at ICU admission, $\mathbf{~ m g} / \mathbf{L}$} \\
\cline { 2 - 3 } & Standardized $\boldsymbol{\beta}$ & $\boldsymbol{P}$ \\
\hline Baseline SCr, umol/L & 0.002 & $<0.001$ \\
SCr at ICU admission, umol/L & 0.004 & $<0.001$ \\
Grade of AKI & 0.183 & $<0.001$ \\
APACHE II score & 0.009 & $<0.001$ \\
Baseline eGFR, ml/min/1.73 m & -0.003 & $<0.001$ \\
Admission type & -0.105 & $<0.001$ \\
CKD, n (\%) & 0.227 & $<0.001$ \\
Previous use of glucocorticoids, n (\%) & 0.092 & 0.003 \\
Cumulative dose of prednisone, mg & 0.0002 & 0.028 \\
Serum albumin, g/L & -0.004 & 0.001 \\
CHD, n (\%) & 0.118 & 0.001 \\
Age & 0.001 & 0.049 \\
Constant & 0.702 (Unstandardized) & $<0.001$
\end{tabular}

APACHE II score Acute Physiology and Chronic Health Evaluation II score; $A K I$ Acute kidney injury; $B M I$ Body mass index; $C K D$ Chronic kidney disease; $C H D$ Coronary heart disease; CHF Chronic heart failure; COPD Chronic obstructive pulmonary disease; DM Diabetes mellitus; eGFR Estimated glomerular filtration rate; ICU Intensive care unit; KDIGO Kidney Disease: Improving Global Outcomes; $n$ Sample size; $\mathrm{SCr}$ Serum creatinine; Mild-AKI: defined as reaching KDIGO stage 1 diagnostic criteria of AKI; Severe-AKI, defined as reaching KDIGO stage 2 or stage 3 diagnostic criteria of AKI

Independent variables included: baseline $\mathrm{SCr}$, $\mathrm{SCr}$ at ICU admission, previous use of glucocorticoids, cumulative dose of prednisone, APACHE II score, grade of AKI, admission type, malignancy, stoke, hypertension, DM, CHD, CHF, COPD, chronic Liver disease, CKD, Albumin at admission, BMl, eGFR, sex, age.

Variables not listed in the table were removed from the stepwise analysis. Adjusted R square 0.776

Numerous previous clinical studies verified the relationship between $\mathrm{sCysC}$ level and glucocorticoids in patients. Cimerman et al. found that sCysC levels were elevated in steroid-independent patients with asthma after 1 week of methylprednisolone treatment [18]. Several studies in renal transplant recipients have also shown that $\mathrm{sCysC}$ levels are correlated with glucocorticoids [26-29, 39]. Risch et al. showed that the level of sCysC are positively correlated with the doses of glucocorticoids [26]. However, their study suggested that glucocorticoids did not affect the detection of impaired renal function by $\mathrm{sCysC}$, as their obtained data indicated that $\mathrm{sCysC}$ was still more accurate than $\mathrm{SCr}$ to assess the renal function in renal transplant patients. Similarly, Pöge et al. found that the highest sCysC levels occurred on day 2 with high-doses of glucocorticoids in renal transplant patients, which suggested changes in $\mathrm{sCysC}$ levels develop fast [27]. Furthermore, Mendiluce et al. noticed that the increase of $\mathrm{sCysC}$ levels coincides with highdoses glucocorticoid treatment in the first 5 days after renal transplantation and then decreased [28]. Such studies suggested that the change of $\mathrm{sCysC}$ levels might be reversible. Some studies further demonstrated that $\mathrm{sCysC}$ could underestimate GFR resulted from this association [26, 39, 45]. Le Bricon et al. suggested that $\mathrm{sCysC}$ underestimated GFR in $14 \%$ in 3 months after renal transplantation [45]. Therefore, $\mathrm{sCysC}$ may be of limited value in assessing renal function in the case of using glucocorticoids due to underestimating of GFR. However, a recent study by Silva et al. did not observe such interference in lupus nephritis patients undergoing glucocorticoid therapy [46]. They speculated that the gene that codifies $\mathrm{CysC}$ was already activated by the induction because lupus nephritis patients used glucocorticoid medication for a long time. Another study had a discrepant conclusion from a study performed by Cimerman et al. in patients with asthma. However, the glucocorticoids treatment is provided by inhalation, but not systemic use [47]. Previous studies also supported that glucocorticoids not only could raise $\mathrm{sCysC}$ levels but also are associated in a dose-dependent manner with increased sCysC $[26,39]$. In the present study, a multiple linear regression analysis showed that glucocorticoid uses were
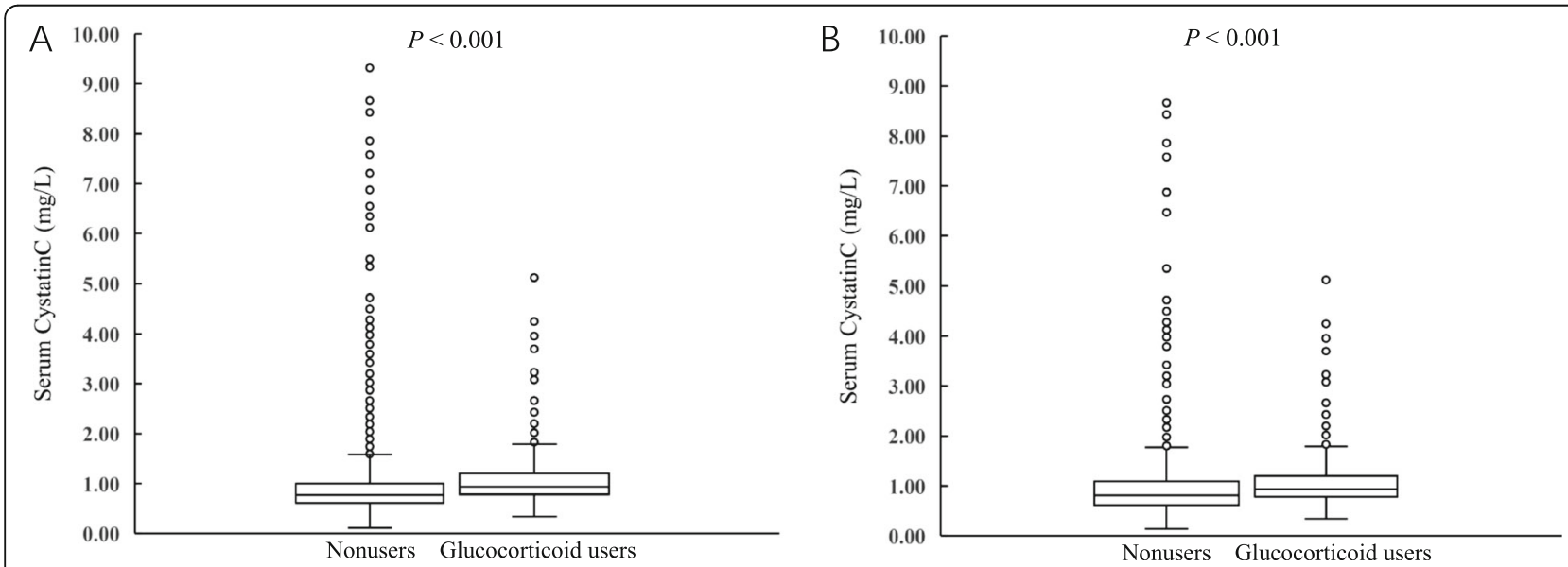

Fig. 2 The median of sCysC levels in patients with and without glucocorticoid treatment. a Glucocorticoid users had higher levels of sCysC before matching $(0.940 \mathrm{mg} / \mathrm{L}$ vs $0.770 \mathrm{mg} / \mathrm{L} ; P<0.001)$. b Glucocorticoid users had higher levels of sCysC after matching $(0.940 \mathrm{mg} / \mathrm{L}$ vs $0.810 \mathrm{mg} / \mathrm{L} ; P<0.001)$ 

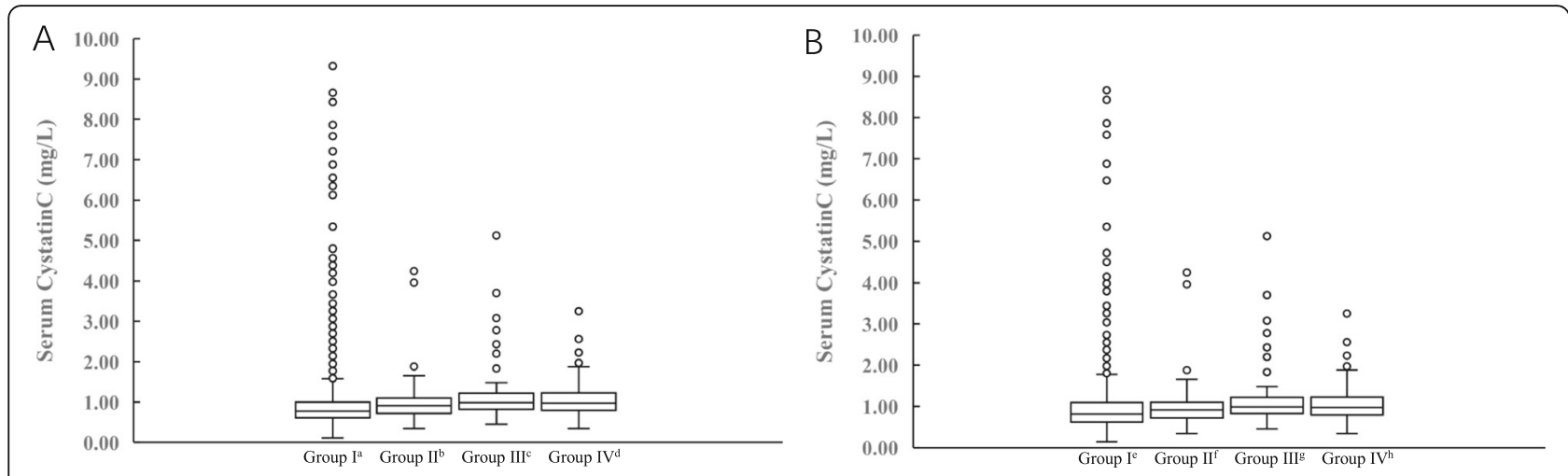

Fig. 3 The concentration of sCysC in different groups. Grouping of standards was according to cumulative doses of glucocorticoids within 5 days before admission to ICU: Group I: non-users; Group II: 0 mg < prednisone $\leq 50$ mg; Group III: 50 mg < prednisone $\leq 150$ mg; Group IV: prednisone $>150$ mg; a Before matching: a: group I vs. group II $P<0.05$, vs. group III $P<0.05$, and vs. group IV $P<0.05$; b: group II vs. group I $P<0.05$, vs. group III $P>0.05$, and vs. group IV $P>0.05$; c: group III vs. group I $P<0.05$, vs. group II $P>0.05$, and vs. group IV $P>0.05$; d: group IV vs. group I $P<0.05$, vs. group II $P>0.05$, and vs. group III $P>0.05$. b After matching: e: group I vs. group $\| P>0.05$, vs. group III $P<0.05$, and vs. group IV $P<$ 0.05; f: group II vs. group | $P>0.05$, vs. group III $P>0.05$, and vs. group IV $P>0.05$; g: group III vs. group $\mid P<0.05$, vs. group ||$P>0.05$, and vs. group IV $P>0.05$; h: group IV vs. group I $P<0.05$, vs. group II $P>0.05$, and vs. group III $P>0.05$

Table 4 Detection of AKI using sCysC by different groups

\begin{tabular}{|c|c|c|c|c|c|c|c|c|c|c|c|c|}
\hline & \multicolumn{4}{|l|}{ Total AKI } & \multicolumn{4}{|c|}{ Established AKI } & \multicolumn{4}{|l|}{ Later-on AKI } \\
\hline & AUC ROC & $95 \% \mathrm{Cl}$ & Cutoff & $P$ & AUC ROC & $95 \% \mathrm{Cl}$ & Cutoff & $P$ & AUC ROC & $95 \% \mathrm{Cl}$ & Cutoff & $P$ \\
\hline \multicolumn{13}{|c|}{ Before matching } \\
\hline Total & $0.769 \pm 0.011$ & $0.753-0.785$ & 1.00 & $<0.001$ & $0.810 \pm 0.017$ & $0.793-0.826$ & 0.93 & $<0.001$ & $0.748 \pm 0.014$ & $0.730-0.765$ & 1.02 & $<0.001$ \\
\hline Group I & $0.769 \pm 0.012$ & $0.752-0.786$ & 1.00 & $<0.001$ & $0.810 \pm 0.018$ & $0.793-0.827$ & 0.93 & $<0.001$ & $0.747 \pm 0.015$ & $0.729-0.765$ & 1.02 & $<0.001$ \\
\hline Group II & $0.733 \pm 0.060$ & $0.613-0.831$ & 1.00 & $<0.001$ & $0.760 \pm 0.086$ & $0.622-0.868$ & 1.04 & 0.003 & $0.714 \pm 0.072$ & $0.580-0.825$ & 1.00 & 0.003 \\
\hline Group III & $0.736 \pm 0.064$ & $0.609-0.840$ & 1.21 & $<0.001$ & $0.781 \pm 0.085$ & $0.631-0.891$ & 1.48 & 0.001 & $0.712 \pm 0.078$ & $0.569-0.829$ & 1.09 & 0.007 \\
\hline Group IV & $0.724 \pm 0.061$ & $0.629-0.805$ & 1.36 & $<0.001$ & $0.779 \pm 0.077$ & $0.676-0.862$ & 0.99 & $<0.001$ & $0.707 \pm 0.074$ & $0.608-0.793$ & 1.64 & 0.005 \\
\hline \multicolumn{13}{|c|}{ After matching } \\
\hline Total & $0.758 \pm 0.015$ & $0.733-0.782$ & 1.09 & $<0.001$ & $0.802 \pm 0.021$ & $0.775-0.827$ & 0.98 & $<0.001$ & $0.732 \pm 0.019$ & $0.704-0.759$ & 1.09 & $<0.001$ \\
\hline Group I & $0.768 \pm 0.017$ & $0.740-0.794$ & 0.95 & $<0.001$ & $0.812 \pm 0.023$ & $0.782-0.839$ & 0.98 & $<0.001$ & $0.740 \pm 0.021$ & $0.709-0.770$ & 0.95 & $<0.001$ \\
\hline Group ॥ & $0.733 \pm 0.060$ & $0.613-0.831$ & 1.00 & $<0.001$ & $0.760 \pm 0.086$ & $0.622-0.868$ & 1.04 & 0.003 & $0.714 \pm 0.072$ & $0.580-0.825$ & 1.00 & 0.003 \\
\hline Group III & $0.736 \pm 0.064$ & $0.609-0.840$ & 1.21 & $<0.001$ & $0.781 \pm 0.085$ & $0.631-0.891$ & 1.48 & 0.001 & $0.712 \pm 0.078$ & $0.569-0.829$ & 1.09 & 0.007 \\
\hline Group IV & $0.724 \pm 0.061$ & $0.629-0.805$ & 1.36 & $<0.001$ & $0.779 \pm 0.077$ & $0.676-0.862$ & 0.99 & $<0.001$ & $0.707 \pm 0.074$ & $0.608-0.793$ & 1.64 & 0.005 \\
\hline
\end{tabular}

95\% CI 95\% Confidence interval; AKI Acute kidney injury; AUC-ROC Area under the receiver operating characteristic curve; ICU Intensive care unit; KDIGO Kidney Disease: Improving Global Outcomes; Established AKI, defined as diagnosis of AKI at ICU admission; Later-onset AKI, indicated no AKI diagnosis at ICU admission but reaching the KDIGO criteria within 1 week after admission;

Grouping of standards was according to cumulative doses of glucocorticoids within 5 days before admission to ICU: Group I: non-users; Group II: 0 mg < prednisone $\leq 50 \mathrm{mg}$; Group III: $50 \mathrm{mg}<$ prednisone $\leq 150 \mathrm{mg}$; Group IV: prednisone $>150 \mathrm{mg}$;

Before matching: Total AKI: AUC of Group I vs. AUC of Group II, $Z=0.588, P=0.556$. AUC of Group I vs. AUC of Group III, $Z=0.507, P=0.612$. AUC of Group I vs. AUC of Group IV, Z = 0.724, $P=0.469$. AUC of Group II vs. AUC of Group III, $Z=-0.034, P=0.973$. AUC of Group II vs. AUC of Group IV, $Z=0.105, P=0.916$. AUC of Group III vs. AUC of Group IV, Z =0.136, $P=0.892$. Established AKI: AUC of Group I vs. AUC of Group II, $Z=0.569, P=0.569$. AUC of Group I vs. AUC of Group III, $Z=0.334, P=0.739$. AUC of Group I vs. AUC of Group IV, $Z=0.392, P=0.695$. AUC of Group II vs. AUC of Group III, $Z=-0.174, P=0.862$. AUC of Group II vs. AUC of Group IV, $Z=-0.165, P=0.869$. AUC of Group III vs. AUC of Group IV, $Z=0.017, P=0.986$. Later-on AKI: AUC of Group I vs. AUC of Group II, $Z=0.449, P=0.654$. AUC of Group I vs. AUC of Group III, $Z=0.441, P=0.660$. AUC of Group I vs. AUC of Group IV, $Z=0.530, P=0.596$. AUC of Group II vs. AUC of Group III, Z =0.019, $P=0.985$. AUC of Group II vs. AUC of Group IV, $Z=0.068, P=0.946$. AUC of Group III vs. AUC of Group IV, $Z=0.047, P=0.963$

After matching: Total AKI: AUC of Group I vs. AUC of Group II, $Z=0.561, P=0.575$. AUC of Group I vs. AUC of Group III, $Z=0.483, P=0.629$. AUC of Group I vs. AUC of Group IV, $Z=0.695, P=0.487$. AUC of Group II vs. AUC of Group III, $Z=-0.034, P=0.973$. AUC of Group II vs. AUC of Group IV, $Z=0.105, P=0.916$. AUC of Group III vs. AUC of Group IV, $Z=0.136, P=0.892$. Established AKI: AUC of Group I vs. AUC of Group II, $Z=0.584, P=0.559$. AUC of Group I vs. AUC of Group III, $Z=0.352, P=0.725$. AUC of Group I vs. AUC of Group IV, $Z=0.411, P=0.681$. AUC of Group II vs. AUC of Group III, $Z=-0.174, P=0.862$. AUC of Group II vs. AUC of Group IV, $Z=-0.165, P=0.869$. AUC of Group III vs. AUC of Group IV, $Z=0.017, P=0.986$. Later-on AKI: AUC of Group I vs. AUC of Group II, $Z=0.347, P=0.729$. AUC of Group I vs. AUC of Group III, $Z=0.347, P=0.729$. AUC of Group I vs. AUC of Group IV, $Z=0.429, P=0.668$. AUC of Group II vs. AUC of Group III, $Z=0.019$, $P=0.985$. AUC of Group II vs. AUC of Group IV, $Z=0.068, P=0.946$. AUC of Group III vs. AUC of Group IV, $Z=0.047, P=0.963$ 
independently correlated with sCysC. After using the statistical approach of propensity score matching, we demonstrated that the elevation of $\mathrm{sCysC}$ levels was related to higher doses of glucocorticoids. However, we did not observe a dose-dependent relationship between the doses of glucocorticoids and sCysC levels. Accumulating evidences suggest that glucocorticoids may have a role in interfering with $\mathrm{sCysC}$ concentrations. Therefore, we can not exclude the possibility that using glucocorticoids may impact the value of $\mathrm{sCysC}$ as a marker of AKI.

As previous and present studies suggested, glucocorticoids induce an elevation of sCysC levels. So, we designed this study to solve the problem of whether the glucocorticoids could impact the performance of sCysC in detecting AKI. The results suggested that glucocorticoids had no statistically significant effect on sCysC in diagnosing and predicting AKI. We suspect that this phenomenon might be a result of the increased sCysC caused by glucocorticoids is only temporary and finite.

This study was not without limitations. First, we used propensity score matching to minimize the effects of potential confounders and selection bias caused by the nonrandom allocation to either the glucocorticoid users or non-users group. Nevertheless, this approach might still have limitations in balancing these factors between the two groups. Second, because our study was not an interventional trial, and we measured sCysC only once at ICU admission, we could not evaluate how long after withholding glucocorticoid therapy sCysC levels are affected. Third, this study did not reveal the rule of change of sCysC cutoff value for AKI detection when patients received glucocorticoid medication. We thought a larger sample size of glucocorticoid users was needed to reveal this rule.

\section{Conclusion}

Glucocorticoids did not impact the performance of sCysC in identifying AKI in critically ill patients, although the levels of sCysC might be slightly affected by glucocorticoid treatment.

\section{Supplementary Information}

The online version contains supplementary material available at https://doi. org/10.1186/s12882-020-02165-1.

\section{Additional file 1:Fig. S1 Performance of sCysC for AKI detection before and after matching. A Performance of sCysC for total AKI detection before and after matching; B Performance of sCysC for established AKI detection before and after matching; C Performance of sCysC for later- onset AKI detection before and after matching; Established AKI, defined as diagnosis of AKI at ICU admission; Later-onset AKI, indicated no AKI diagnosis at ICU admission but reaching the KDIGO criteria within 1 week after admission; AKI: Acute kidney injury; ICU: Intensive care unit; KDIGO: Kidney Disease: Improving Global Outcomes;}

\section{Abbreviations}

95\% Cl: 95\% Confidence interval; AKI: Acute kidney injury; APACHE II: Acute physiology and chronic health evaluation II score; AUC: Area under the receiver operating characteristic curve; BMI: Body mass index; CHD: Coronary heart disease; CHF: Chronic heart failure; CKD: Chronic kidney disease; COPD: Chronic obstructive pulmonary disease; CysC: Cystatin C; DM: Diabetes mellitus; eGFR: Estimated glomerular filtration rate; EPI: Epidemiology collaboration; ESRD: End-stage renal disease; GFR: Glomerular filtration rate; ICU: Intensive care unit; KDIGO: Kidney disease: improving global outcomes; n: Sample size; ROC: Receiver operating characteristic curve; RRT: Renal replacement therapy; SCr: Serum creatinine; sCysC: Serum cystatin C

\section{Acknowledgements}

The authors thank all the doctors, nurses, technicians, and patients at the Guangdong Provincial People's Hospital for their dedication in the study.

\section{Authors' contributions}

CBC, SLL MS, and YPB equally contributed to the design of the research. SLL and YPB equally contributed to analyze and interpret the data. CBC and MS contributed to the conception of the research and critically revised the manuscript. SLL, MS, YPB, YJD, MXF, JXL, YJW, WYP, YTH, HF, and HDZ performed the research and collected data. All authors contributed to the acquisition and analysis of the data, drafted the manuscript, and agree to be fully accountable for ensuring the integrity and accuracy of the work. All authors read and approved the final manuscript.

\section{Funding}

The study was supported by Chunbo Chen's research grants from the National Natural Science Foundation of China [81671963], the Science and Technology Planning Project of Guangdong Province (Key Program) [2014B020212023], the Guangzhou Livelihood Science and Technology Program [201803010058], and Yujun Deng's research grant from the Science and Technology Planning Project of Guangdong Province [2016A020215129].

\section{Availability of data and materials}

The datasets generated and/or analyzed during this study are not publicly available, owing to currently ongoing research studies, but the data are available from the corresponding author on reasonable request.

\section{Ethics approval and consent to participate}

The current study received the approval of ethics committee of the Guangdong Provincial People's Hospital. The ethics committee of the Guangdong Provincial People's Hospital supervised the study, including the study design, protocol, ethical issue, and data and sample collection. Written informed consent was obtained from each patient or from the appropriate guardian.

\section{Consent for publication}

Not applicable. No individual personal data are included in the study. All patients provided necessary consent to participate in the present study.

\section{Competing interests}

The authors declare that they have no competing interests.

\section{Author details}

'Department of Intensive Care Unit of Cardiovascular Surgery, Guangdong Cardiovascular Institute, Guangdong Provincial People's Hospital, Guangdong Academy of Medical Sciences, 96 Dongchuan Road, Guangzhou 510080, Guangdong Province, People's Republic of China. ${ }^{2}$ Department of Critical Care Medicine, Guangdong Provincial People's Hospital, Guangdong Academy of Medical Sciences, 106 Zhongshan Er Road, Guangzhou 510080, Guangdong Province, People's Republic of China. ${ }^{3}$ The Second School of Clinical Medicine, Southern Medical University, Guangzhou, China.

${ }^{4}$ Department of Critical Medicine, Peking University Third Hospital, No.49, Huayuan Rd., Haidian District, Beijing 100191, People's Republic of China. ${ }^{5}$ Center of Scientific Research, Maoming People's Hospital, 101 Weimin Road, Maoming 525000, Guangdong Province, People's Republic of China. ${ }^{6}$ Department of Critical Care Medicine, Maoming People's Hospital, 101 Weimin Road, Maoming 525000, Guangdong Province, People's Republic of China. ${ }^{7}$ Department of Oncology, Maoming People's Hospital, 101 Weimin Road, Maoming 525000, Guangdong Province, People's Republic of China. 
Received: 22 September 2020 Accepted: 9 November 2020

Published online: 27 November 2020

\section{References}

1. RPd S, ARS C, LAB P, Ronco C, Macedo E. An epidemiologic overview of acute kidney injury in intensive care units. Rev Assoc Méd Bras. 2019;65:1094-101.

2. Negi S, Koreeda D, Kobayashi S, Yano T, Tatsuta K, Mima T, et al. Acute kidney injury: epidemiology, outcomes, complications, and therapeutic strategies. Semin Dial. 2018;31(5):519-27.

3. Hoste EAJ, Bagshaw SM, Bellomo R, Cely CM, Colman R, Cruz DN, et al. Epidemiology of acute kidney injury in critically ill patients: the multinational AKI-EPI study. Intensive Care Med. 2015;41(8):1411-23.

4. Folkestad T, Brurberg KG, Nordhuus KM, Tveiten CK, Guttormsen AB, Os I, et al. Acute kidney injury in burn patients admitted to the intensive care unit: a systematic review and meta-analysis. Crit Care. 2020;24(1):2.

5. Jo SK, Rosner MH, Okusa MD. Pharmacologic treatment of acute kidney injury: why drugs haven't worked and what is on the horizon. Clin J Am Soc Nephrol. 2007;2(2):356-65.

6. Pakula AM, Skinner RA. Acute kidney injury in the critically ill patient:a current review of the literature. J Intensive Care Med. 2016;31(5):319-24.

7. Randers E, Erlandsen EJ. Serum cystatin $C$ as an endogenous marker of the renal function--a review. Clin Chem Lab Med. 1999:37(4):389-95.

8. Ostermann M, Joannidis M. Acute kidney injury 2016: diagnosis and diagnostic workup. Crit Care. 2016;20(1):299.

9. Teo $\mathrm{SH}$, Endre ZH. Biomarkers in acute kidney injury (AKI). Best Pract Res Clin Anaesthesiol. 2017:31(3):331-44

10. Dharnidharka VR, Kwon C, Stevens G. Serum cystatin C is superior to serum creatinine as a marker of kidney function: a meta-analysis. Am J Kidney Dis. 2002:40(2):221-6.

11. Laterza OF, Price CP, Scott MG. Cystatin C: an improved estimator of glomerular filtration rate? Clin Chem. 2002;48(5):699-707.

12. Herget-Rosenthal S, Marggraf G, Husing J, Goring F, Pietruck F, Janssen O, et al. Early detection of acute renal failure by serum cystatin C. Kidney Int. 2004;66(3):1115-22.

13. Charlton JR, Portilla D, Okusa MD. A basic science view of acute kidney injury biomarkers. Nephrol Dial Transplant. 2014;29(7):1301-11.

14. Deng Y, Chi R, Chen S, Ye H, Yuan J, Wang L, et al. Evaluation of clinically available renal biomarkers in critically ill adults: a prospective multicenter observational study. Crit Care. 2017;21(1):46.

15. Deng Y, Yuan J, Chi R, Ye H, Zhou D, Wang S, et al. The incidence, risk factors and outcomes of postoperative acute kidney injury in neurosurgical critically ill patients. Sci Rep. 2017;7(1):4245.

16. Deng Y, Ma J, Hou Y, Zhou D, Hou T, Li J, et al. Combining serum Cystatin C and urinary $\mathrm{N}$-acetyl-Beta-D-Glucosaminidase improves the precision foracute kidney injury diagnosis after resection of intracranial spaceoccupying lesions. Kidney Blood Press Res. 2020;45(1):142-56.

17. Manetti L, Genovesi M, Pardini E, Grasso L, Lupi I, Linda Morselli L, et al. Early effects of methylprednisolone infusion on serum cystatin $C$ in patients with severe Graves' ophthalmopathy. Clin Chim Acta. 2005;356(1):227-8.

18. Cimerman N, Brguljan PM, Krašovec M, Šuškovič S, Kos J. Serum cystatin C, a potent inhibitor of cysteine proteinases, is elevated in asthmatic patients. Clin Chim Acta. 2000;300(1):83-95.

19. Zhang D, Gao L, Ye H, Chi R, Wang L, Hu L, et al. Impact of thyroid function on cystatin $C$ in detecting acute kidney injury: a prospective, observational study. BMC Nephrol. 2019;20(1):41.

20. Deng $Y$, Wang $L$, Hou $Y, M a J$, Chi R, Ye H, et al. The influence of glycemic status on the performance of cystatin $C$ for acute kidney injury detection in the critically ill. Ren Fail. 2019;41(1):139-49.

21. Stevens LA, Schmid CH, Greene T, Li L, Beck GJ, Joffe MM, et al. Factors other than glomerular filtration rate affect serum cystatin $C$ levels. Kidney Int. 2009:75(6):652-60

22. Royakkers AA, Korevaar JC, van Suijlen JD, Hofstra LS, Kuiper MA, Spronk PE, et al. Serum and urine cystatin $C$ are poor biomarkers for acute kidney injury and renal replacement therapy. Intensive Care Med. 2011;37(3):493-501.

23. Bjarnadottir M, Grubb A, Olafsson I. Promoter-mediated, dexamethasoneinduced increase in cystatin C production by HeLa cells. Scand J Clin Lab Invest. 1995;55(7):617-23.

24. Munoz J, Soblechero P, Duque FJ, Macias-Garcia B, Ruiz P, Zaragoza C, et al. Effects of Oral prednisone administration on serum Cystatin $C$ in dogs. J Vet Intern Med. 2017;31(6):1765-70.
25. Zhu X-R, Ge N, Wang Y, Zhai J-L, Liu C. Corticosteroids significantly increase cystatin $\mathrm{C}$ levels in the plasma by promoting cystatin $\mathrm{C}$ production in rats. Ren Fail. 2019;41(1):698-703.

26. Risch $L$, Herklotz R, Blumberg A, Huber AR. Effects of glucocorticoid immunosuppression on serum Cystatin C concentrations in renal transplant patients. Clin Chem. 2001;47(11):2055-9.

27. Poge U, Gerhardt T, Bokenkamp A, Stoffel-Wagner B, Klehr HU, Sauerbruch T, et al. Time course of low molecular weight proteins in the early kidney transplantation period-influence of corticosteroids. Nephrol Dial Transplant. 2004;19(11):2858-63.

28. Mendiluce A, Bustamante J, Martin D, Santos M, Bustamante R, Pascual P, et al. Cystatin $C$ as a marker of renal function in kidney transplant patients. Transplant Proc. 2005;37(9):3844-7.

29. Geramizadeh B, Azarpira N, Ayatollahi M, Rais-Jalali G-A, Aghdai M, Yaghoobi R, et al. Value of serum cystatin $C$ as a marker of renal function in the early post kidney transplant period. Saudi J Kidney Dis Transplant. 2009:20(6):1015-7.

30. von Elm E, Altman DG, Egger M, Pocock SJ, Gøtzsche PC, Vandenbroucke $J P$. The strengthening the reporting of observational studies in epidemiology (STROBE) statement: guidelines for reporting observational studies. PLOS Med. 2007:4(10):e296.

31. Bossuyt PM, Reitsma JB, Bruns DE, Gatsonis CA, Glasziou PP, Irwig LM, et al. Toward complete and accurate reporting of studies of diagnostic accuracy: the STARD initiative. Acad Radiol. 2003;10(6):664-9.

32. Levey AS, Stevens LA, Schmid CH, Zhang YL, Castro AF 3rd, Feldman HI, et al. A new equation to estimate glomerular filtration rate. Ann Intern Med. 2009;150(9):604-12.

33. Khwaja A. KDIGO clinical practice guidelines for acute kidney injury. Nephron Clin Pract. 2012;120(4):c179-84.

34. McCullough PA, Shaw AD, Haase M, Bouchard J, Waikar SS, Siew ED, et al. Diagnosis of acute kidney injury using functional and injury biomarkers: workgroup statements from the tenth acute Dialysis quality initiative consensus conference. Contrib Nephrol. 2013;182:13-29.

35. Endre ZH, Walker RJ, Pickering JW, Shaw GM, Frampton CM, Henderson SJ, et al. Early intervention with erythropoietin does not affect the outcome of acute kidney injury (the EARLYARF trial). Kidney Int. 2010;77(11):1020-30.

36. Basu RK, Wang Y, Wong HR, Chawla LS, Wheeler DS, Goldstein SL. Incorporation of biomarkers with the renal angina index for prediction of severe AKI in critically ill children. Clin J Am Soc Nephrol. 2014;9(4):654-62

37. Meikle AW, Tyler FH. Potency and duration of action of glucocorticoids: effects of hydrocortisone, prednisone and dexamethasone on human pituitary-adrenal function. Am J Med. 1977;63(2):200-7.

38. Ragab SM, Helwa MA, Khalaf AA. Serum cystatin C and microalbuminuria in children with immune thrombocytopenia under short course of corticosteroids. Egypt Pediatr Assoc Gaz. 2015;63(2):39-45.

39. Gruev T, Chakalarovski K, Stojceva-Taneva O, Grueva A, Trenceva K. Effects of glucocorticoid immunosuppression on serum cystatin C levels. J Med Biochem. 2009;28(3):191-6.

40. Abbink FCH, Laarman CARC, Braam KI, van Wijk JAE, Kors WA, Bouman AA, et al. Beta-trace protein is not superior to cystatin C for the estimation of GFR in patients receiving corticosteroids. Clin Biochem. 2008;41(4):299-305.

41. Borges RL, Hirota AH, Quinto BMR, Ribeiro AB, Zanella MT, Batista MC. Is Cystatin $\mathrm{C}$ a useful marker in the detection of diabetic kidney disease? Nephron Clin Pract. 2010;114(2):c127-34.

42. Fricker $M$, Wiesli $P$, Brändle $M$, Schwegler $B$, Schmid C. Impact of thyroid dysfunction on serum cystatin C. Kidney Int. 2003;63(5):1944-7.

43. Song T, Jia Z, Guo X, Zhao H, Bao W, Han D, et al. Does hepatic impairment influence renal function parameters in liver cirrhosis? J Transl Int Med. 2018;6(2):90-2

44. Ghane Shahrbaf F, Assadi F. Drug-induced renal disorders. J Renal Inj Prev. 2015;4(3):57-60

45. Le Bricon T, Thervet E, Froissart M, Benlakehal M, Bousquet B, Legendre C, et al. Plasma Cystatin $C$ is superior to $24-h$ Creatinine clearance and plasma Creatinine for estimation of glomerular filtration rate 3 months after kidney transplantation. Clin Chem. 2000;46:1206-7.

46. Silva MV, Moscoso Solorzano G, Nishida SK, Kirsztajn GM. Are serum cystatin C levels infl uenced by steroid doses in lupus nephritis patients? J Bras Nefrol. 2011;33.

47. El-Esawy NM, Morad MA, Abdin AA. Urinary Creatinine and serum Cystatin C in asthmatic children treated with inhaled corticosteroid and Beta 2 agonist Alex J Pediatr. 2005;19(2):231.

\section{Publisher's Note}

Springer Nature remains neutral with regard to jurisdictional claims in published maps and institutional affiliations. 